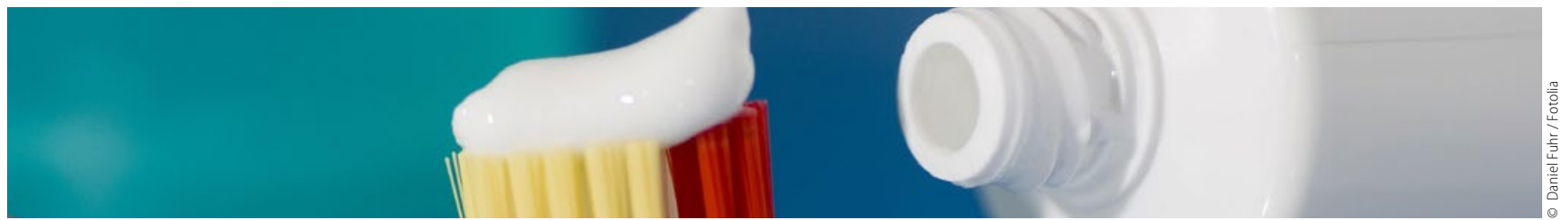

Resininfiltration, Fluoridierung, Nano-Hydrylapatitbehandlung

\title{
Fortschritte bei der Behandlung initialer Karies
}

In den vergangenen Jahren haben sich die Methoden zur Behandlung dentaler Karies grundsätzlich geändert. Die ersten Anzeichen einer Demineralisation sind weiße Schmelzflecken. Bei einer kieferorthopädischen Behandlung treten diese weißen Flecken regelmäßig auf. Andere prädisponierende Faktoren sind beispielsweise eine schlechte Mundhygiene oder eine Hypomineralisation des Speichels.

Der Schmelz dieser weißen Flecken besitzt keine intakte Oberfläche. Unter elektronenmikroskopischer Betrachtung der Kristallstruktur des Schmelzes ist dieser verändert und der Gehalt an Kalzium und Phosphat vermindert.

Zur Therapie sind die Optimierung der Mundhygiene und die Förderung der Remineralisation notwendig. Für die Beseitigung der weißen Flecken sind verschiedene Methoden etabliert worden: zum einen das konventionelle Bonding der Läsion oder die Resininfiltration, aber auch die Remineralisation des Schmelzes mit Hilfe einer topischen Anwendung von Fluoriden und amorphem Kalziumphosphat, das sogenannte ACP. Dieses ACP ist ein Vorläufer des Hydroxylapatits und fördert die Remineralisation des Schmelzes sowie die Aufnahme von Fluor. Insbesondere im sauren Milieu ist der Kalzium- und Phosphatgehalt in diesen Läsionen verglichen mit der Umgebung stark erhöht.

Ein alternativer Ansatz zur Behandlung dieser initialen Schmelzläsionen ist die Infiltration der Schmelzporen mit lichthärtendem, dünnflüssigem Komposit. Diese Methode stoppt nicht nur das Fortschreiten der Bakteriensäuren, sondern stärkt auch die Härte des Schmelzes.

\section{Freie Oberflächenenergie ist von Bedeutung}

Die Adhäsion der Bakterien auf der Schmelzoberfläche stellt die initiale Phase der Gingivitis und der Sekundärkaries dar, so dass bei der Resininfiltration auch die Oberflächenrauigkeit und freie Oberflächenenergie von Bedeutung sind.

Im Rahmen von In-vitro-Studien konnte gezeigt werden, dass die Schmelzoberfläche mit Hilfe der Resininfiltration gestärkt wird und die Bakterienadhäsion wegen der glatten Zahnoberfläche vermindert werden konnte. Eine andere Forschergruppe verglich die Methode der Schmelzinfiltration mit der Fluoridbehandlung beziehungsweise Nanohydroxylapatitbehandlung. Im direkten Vergleich konnte die Resininfiltration die härteste Oberfläche generieren. Die Fluoridbehandlung reduzierte die Bakterienadhäsion am effektivsten. Die Behandlung der initialen Demineralisationsläsionen mit Nanohydroxylapatit führte zu einer härteren Schmelzoberfläche als bei den fluoridbehandelten Zähnen sowie zu einer geringeren Bakterienadhäsion als bei den resinbehandelten Zähnen.

\section{Quellen}

1. M. Aziznezhad et al. (2017) Comparison oft he effect of resin infiltration, fluorid vanish, and nano-hydroxy apatite paste on surface hardness an dstreptococcus mtans adhesion to arificial enamel lesion. 9(3):39343942

2. S. Arslan et al. (2015) Effect of resin infiltration on enamel surface properties and Streptococcus mutans adhesion to artificial enamel lesions

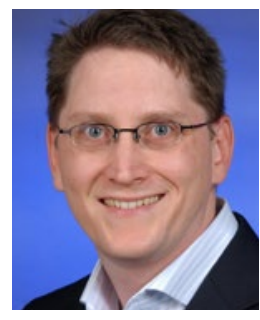

Prof. Dr. Dr. Felix P. Koch

Autor des Wissenschaftlichen Infodienstes, Lehrbeauftragter der Uniklinik Frankfurt, Praxisklinik für Mund-, Kiefer- und plastische Gesichtschirurgie in Wiesbaden. Mail:wid@fvdz.de 